\title{
Trichinosis Imitating an Inflammatory Systematic Disease
}

\author{
Maria Kosmidou',* ${ }^{1}$, Georgios Papamichail', Eleftherios Klouras' ${ }^{1}$ Konstantinos Tsamis², loannis Sarmas², \\ Iro Rapti ${ }^{1}$, Haralampos Milionis ${ }^{1}$ \\ ${ }^{1}$ First Division of Internal Medicine, Faculty of Medicine, University of loannina, loannina 45110, Greece; ${ }^{2}$ Department of Neurology, \\ Faculty of Medicine, University of loannina, Ioannina 45110, Greece
}

\begin{abstract}
Trichinellosis (trichinosis) is a parasitic infection caused by nematodes of the genus Trichinella. Pigs are the most common source of human infection. We describe a case of a 47-year-old woman presented with a wide range of intermittent symptoms including prolonged fever, dry cough, diarrhea, rash, myalgias and arthralgias. The patient was attended by physicians with various medical specialties such as dermatologists, rheumatologists and allergiologists, but they did not establish a certain diagnosis because of the gradual onset of symptoms, raising the suspicion of a systematic disease. After extensive work up, the diagnosis of trichinosis was established with femoral muscle biopsy compatible with inflammatory myopathy of parasitic etiology with trichinosis to be the predominant diagnosis. Despite the significant delay of diagnosis for almost three months, patient was treated successfully with no further complications. Trichinellosis is a food-borne treatable infection. Preventive measures include community education especially in zones where parasite prevalence is increased, improvement of farming and cooking techniques.
\end{abstract}

Key words: Trichinosis, muscle biopsy, inflammatory systematic disease

\section{INTRODUCTION}

Trichinellosis (trichinosis) is a parasitic infection caused by nematodes of the genus Trichinella. Pigs are the most common source of human infection, although a number of other animals are also epidemiologically important hosts. Consumption of undercooked meat is the principal way of transmission. Trichinellosis has been reported worldwide but its prevalence is particularly high in North America, China, Thailand, Mexico, Argentina, Bolivia, Romania, and other parts of Central Europe [1-6].

For heavy infections, 2 clinical stages may be observed [3-5]. The intestinal stage occurs between the second and seventh day after ingestion, when encysted larvae are liberated from the infected meat. This stage may be asymptomatic or symptoms including abdominal pain, nausea, vomiting, and diarrhea may be present [3-5].

The muscle stage develops after the first week of ingestion and indicates the hematogenous spread of adult-derived larvae

\footnotetext{
- Received 5 April 2021, revised 4 August 2021, accepted 12 August 2021.

*Corresponding author (mkosmid@uoi.gr)

(C) 2021. Korean Society for Parasitology and Tropical Medicine

This is an Open Access article distributed under the terms of the Creative Commons

Attribution Non-Commercial License (https://creativecommons.org/licenses/by-nc/4.0) which permits unrestricted non-commercial use, distribution, and reproduction in any

medium, provided the original work is properly cited.
}

from intestine and their entrance in skeletal muscle. The pain can be so intense, leading to movement difficulty, including breathing or moving the tongue. Less common manifestations include macular or urticarial rash, headache, cough, dyspnea, and dysphagia [3-6].

\section{CASE DESCRIPTION}

A 47-year-old woman of urban origin presented in our hospital with a wide range of symptoms during the last 3 weeks, including prolonged fever, dry cough, diarrhea, rash and arthralgias. Fever was the first symptom, accompanied by diarrhea, lasting for 4 days. There was a 2-day recession of symptoms and then a relapse of fever occurred with a simultaneous rash spreading all over the trunk and extremities. Patient referred to the emergency department of another hospital and after administration of corticosteroids, antihistamine drugs and antibiotics (Methylprednisone $32 \mathrm{mg} / \mathrm{d}$, dimetindene 2 $\mathrm{mg}$ and ceftriaxone $2 \mathrm{gr} / \mathrm{d}$ ) for a week, a significant improvement of symptoms was noticed. After completing this medication, a second relapse of symptoms with an onset of lips edema and dry cough was aroused leading patient to a dermatologist and then to our hospital.

On her admission, patient was febrile with a mild erythema on her both cheeks, a maculopapular rash on her back, myal- 
gias and livedo reticularis of lower extremities. The rest of physical examination was unrevealing. Her medical history was unremarkable, while her mother had been diagnosed with Sjogren syndrome and both parents had a history of coronary artery disease at an early age.

The laboratory examination showed: leukocytosis with eosinophilia up to $1,690 / \mu l$, increased levels of C-reactive protein (CRP): $75 \mathrm{mg} / \mathrm{dl}$, (normal value: $<6 \mathrm{mg} / \mathrm{dl}$ ), creatinine phosphokinase (CPK): 1,200 mg/dl (normal value: 30-190 mg/dl), aldolase: $14.5 \mathrm{U} / \mathrm{L}$ (normal values: 1-7.5 U/L), lactate dehydrogenase (LDH): $680 \mathrm{mg} / \mathrm{dl}$ (normal values: $140-280 \mathrm{mg} / \mathrm{dl}$ ), suggesting a possible muscular tissue damage.

Taking into consideration that the patient's symptoms were persistent myalgias, arthralgias, recurrent rash and the main laboratory findings were the elevated inflammatory markers, the deferential diagnosis was restricted to the spectrum of autoimmune inflammatory diseases like systematic lupus erythematosus, rheumatoid arthritis or myositis. Drug reaction with eosinophilia and systemic symptoms (DRESS) syndrome was also considered in the deferential diagnosis, given that there was a history of non-steroidal anti-inflammatory drugs intake.

The patient underwent an extensive diagnostic work-up including an abdomen ultrasound, a computer tomography of thorax and a color echocardiogram due to high sensitive cardiac troponine increased levels (hs-cTn): $117 \mathrm{ng} / \mathrm{L}$, without a significant outcome as no mass in organs, effusion or cardiac valve vegetation and myocardial dysfunction was depicted. The neurologic examination revealed mild weakness of the upper and lower extremities and electroneurographic testing was normal. Electromyography (EMG) showed fibrillations and positive waves in both peripheral and proximal muscles, indicative of an irritative myopathy. Then we proceeded with a femoral muscle biopsy after 3 months from the onset of her symptoms.

Patient received methylprednisolone $16 \mathrm{mg}$ orally per day, and defervesence achieved with a simultaneous recession of CRP and CPK levels and eosinophils count, as expected after corticosteroids' initiation. She was discharged with recommendation of corticosteroids continuation until the results of muscle biopsy would be available.

The histological estimation the femoral muscular tissue was compatible with inflammatory myopathy of parasitic etiology with trichinosis to be the predominant diagnosis. Since central neural system involvement characterize the severe disease $[3,4,6]$, patient underwent a brain MRI without any pathological findings (Fig. 1). Patient admitted the consumption of un-
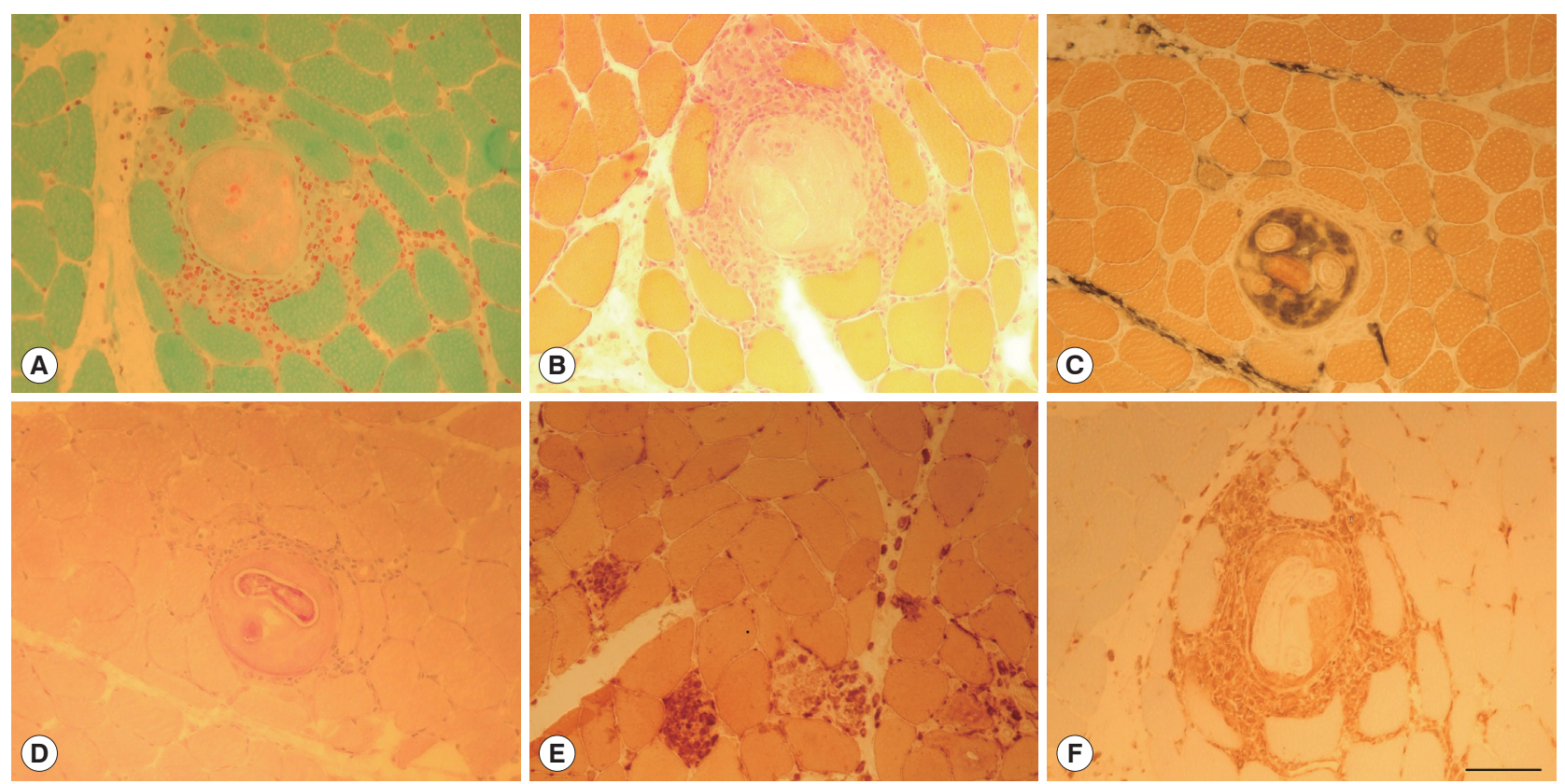

Fig. 1. Biopsy of the quadriceps muscle. Gomori trichrome stain (A) and Hematoxylin \& Eosin stain (B) reveal cysts surrounded by inflammatory cells. The material encapsulated in the cysts stain with Alkaline Phosphatase (C) and larvae stain strongly with Periodic Acid Schiff (PAS) staining (D). Necrotic muscle fibers are sporadically observed, as well as muscle fibers replaced by large histiocytic cells stained with Esterase staining (E). Inflammatory cells surrounding cysts and invading muscle fibers are macrophages CD68 immunopositive (F). Scale bar in (F) $100 \mu \mathrm{m}$ (valid for $(A-F)$ ). 
dercooked wild boar minced meat a week before the onset of symptom: accidently while she was preparing the food she tasted the minced meat. No one else within the family was infected.

Albendazole $400 \mathrm{mg}$ bid for 14 days was initiated as an anthelmintic agent, leading to a complete clinical and laboratory response even from the first week of its administration.

\section{DISCUSSION}

As we described above, in the case of this 47-year-old woman there were 2 distinct stages related to her clinical manifestations which are completely compatible with the 2 common stages of trichinella pathogenesis [3-5]. In the first stage, woman experienced fever and diarrhea (intestinal stage) and in the second stage, she suffered from myalgias (the muscle stage) with the appearance of a rash, a rare manifestation of trichinosis. These stages experienced form the patient in periods which are in an absolute accordance to life cycle stages of the parasite [3-5]. On the other hand, it was difficult to determine the source of the transmission, until the last follow up when she mentioned the consumption of a possible undercooked wild boar minced meat.

In this case, the clinical manifestations of the distinct stages of the infection led to misdiagnosis, while the EMG findings and the muscle biopsy, led to diagnosis of Trichinellosis. It is of great importance the acknowledgment of the course of thrichinellosis, in order to establish diagnosis as soon as possible.

The most important delay was that the patient referred to a dermatologist, allergiologist and rheumatologist whom they did not establish a certain diagnosis because of the gradual onset of symptoms which raised the suspicion of a systematic disease. Despite the fact that this situation provoked a significant delay in patient diagnosis and treatment there were no further complications.

Trichinellosis is a food-borne treatable infection. Preventive measures include community education especially in zones where parasite prevalence is increased, improvement of farming and cooking techniques to inactivate Trichinella larvae in meat [1-6].

\section{CONFLICT OF INTERSET}

The authors report no potential conflict of interest

\section{REFERENCES}

1. Diaz JH, Warren RJ, Oster MJ. The disease ecology, epidemiology, clinical manifestations, and management of trichinellosis linked to consumption of wild animal meat. Wilderness Environ Med 2020; 31: 235-244. https://doi.org/10.1016/j.wem.2019.12.003

2. Ribicich MM, Fariña FA, Aronowicz T, Ercole ME, Bessi C, Winter M, Pasqualetti MI. A review on Trichinella infection in South America. Vet Parasitol 2020; 285: 109234. https://doi.org/10.1016/j.vetpar. 2020.109234

3. Gould SE. Clinical manifestations. In Gould SE ed, Trichinosis in Man and Animals. Springfield, USA. Charles C Thomas. 1970, pp 269.

4. MacLean JD, Poirier L, Gyorkos TW, Proulx JF, Bourgeault J, Corriveau A, Illisituk S, Staudt M. Epidemiologic and serologic definition of primary and secondary trichinosis in the Arctic. J Infect Dis 1992; 165: 908-912. https://doi.org/10.1093/infdis/165.5.908

5. Neghina R, Neghina AM. Reviews on trichinellosis (IV): hepatic involvement. Foodborne Pathog Dis 2011; 8: 943-948. https:// doi.org/10.1089/fpd.2011.0861

6. Bruschi F, Chiumiento L. Trichinella inflammatory myopathy: host or parasite strategy? Parasit Vectors 2011; 4: 42. https://doi. org/10.1186/1756-3305-4-42 
